\title{
Estrategias de enseñanza y aprendizaje de la asignatura de anatomía humana de los estudiantes del tercer ciclo, Facultad de Ciencias Médicas Universidad Nacional "Santiago Antúnez de Mayolo" - Huaraz, 2018
}

\section{Teaching and learning strategies of the course of human anatomy of students of the iii cycle, Faculty of Medical Sciences Universidad National "Santiago Antúnez de Mayolo" - Huaraz, 2018}

Herlinda Irma Valentín Vargas, Abelardo Rodolfo Campana Concha

\begin{abstract}
RESUMEN
Objetivo: Estudiar el nivel de relación entre las variables estrategias de enseñanza y el aprendizaje de la asignatura de anatomía humana de estudiantes del III Ciclo, Facultad de Ciencias Médicas Universidad Nacional "Santiago Antúnez de Mayolo" - Huaraz, 2018. Material y Métodos: La investigación tiene un enfoque cuantitativo de diseño correlacional. Se utilizó el programa SPSS, asimismo se siguió el procedimiento estadístico de Rho de Spearman. Los instrumentos fueron aplicados a una muestra de 83 estudiantes. Resultado: La hipótesis general estableció una correlación muy buena de $\mathrm{Rho}=0,845$ que establece la perspectiva de los estudiantes, las estrategias de enseñanza son a veces aplicado por los docentes incidiendo en las calificaciones regulares del curso de Anatomía Humana. Las hipótesis específicas 1, 2, 3 y 4 hallaron una correlación moderada de $\mathrm{Rho}=0,497$, Rho $=0,589$, $\mathrm{Rho}=0,595$ y $\mathrm{Rho}=0,581$, respectivamente; se identifica la Activación de conocimientos previos, Orientación y guía de contenidos, Codificación y Organización de la información que está relacionada con el Aprendizaje de la Asignatura de la Anatomía Humana; mientras la hipótesis específica 5 encontró una correlación buena de Rho $=0,728$, entiende que los conocimientos previos y nuevos repercute en los aprendizajes del curso anteriormente mencionado. Conclusiones: La importancia y la necesidad de garantizar aprendizajes, se debe al establecimiento idóneo de una metodología de enseñanza, sin embargo, se necesita un seguimiento constante para identificar si se están alcanzando los objetivos académicos para mejorar aprendizajes, los cuales deberán ser reflejado en sus calificaciones.
\end{abstract}

Palabras clave: Estrategias de enseñanza, aprendizaje, anatomía humana, conocimientos previos, guía de contenidos, codificación, organización.

\begin{abstract}
Objective: Study the level of relationship between the variables teaching strategies and learning of the subject of human anatomy of students of the III Cycle, Faculty of Medical Sciences National University "Santiago Antúnez de Mayolo" - Huaraz, 2018. Material and Methods: Research has a quantitative approach to correlational design. The SPSS program was used, also the statistical procedure of Spearman's Rho was followed. The instruments were applied to a sample of 83 students. Result: The general hypothesis established a very good correlation of Rho $=0,845$ that establishes the students' perspective, the teaching strategies are sometimes applied by the teachers influencing the regular grades of the Human Anatomy course. Specific hypotheses 1, 2, 3 and 4 found a moderate correlation of Rho $=0.497$, Rho $=0.589$, Rho $=0.595$ and Rho $=0.581$, respectively; the Activation of previous knowledge, Orientation and guide of contents, Codification and Organization of the information that is related to the Learning of the Subject of the Human Anatomy is identified; While the specific hypothesis 5 found a good correlation of Rho $=0.728$, it understands that previous and new knowledge affects the learning of the aforementioned course. Conclusions: The importance and the need to guarantee learning, is due to the suitable establishment of a teaching methodology, however, a constant follow-up is needed to identify if the academic objectives are being achieved to improve learning, which should be reflected in their ratings.
\end{abstract}

Keywords: Teaching strategies, learning, human anatomy, previous knowledge, content guide, coding, organization.

\section{INTRODUCCIÓN}

EI INEI (2015) destaca que el nivel ocupacional universitario por áreas de estudio, en donde $16,5 \%$ corresponden a las especialidades de Ciencias Administrativas y Comerciales, $13,4 \%$ a la especialidad de Ciencias de la Salud, $12,1 \%$ a Ciencias Económicas y Contables, 10,3\% Ingeniería Industrial y Producción y $9,1 \%$ a Derecho, Ciencias Políticas y Jurídicas. Es decir, las carreras relacionadas a la salud es la segunda área con más demanda, por lo cual se evidencia mayor grado de competitividad, obligando así a las instituciones educativas a formar buenos profesionales, contando con las herramientas necesarias para ello.

Por otro lado, entre los universitarios destaca actividades culturales, seguido del servicio de biblioteca, representados en el $52,5 \%$ y $50,3 \%$ lo consideran de bueno respectivamente, sin embargo, aún no consiguen el nivel excelente, otro punto en cuestión es que en la lista no están los servicios académicos adicionales que fortalezcan sus conocimientos como cursos de capacitación, nivelación, actualización, etc.

Con respecto a los docentes, principales responsables de las estrategias de enseñanza, el INEI (2015), a nivel nacional, $57,3 \%$ de egresados perciben la calidad docente como buena y $18,3 \%$, existe un $24,4 \%$ que no comparte dicha afirmación.
A perspectiva de los encuestados de la universidad peruana, refiere a la infraestructura universitaria como destacada y los egresados universitarios califican como buenos o excelentes; los auditorios $52,8 \%$ señalan como buena infraestructura y $13,9 \%$ opinan excelente; las aulas calificadas como buenas $49,7 \% ; 51,6 \%$ de egresados de universidades públicas consideran los auditorios como la mejor; para el 56,0\% las instalaciones sanitarias de la universidades privadas.

A nivel nacional, $57,3 \%$ de egresados refieren que la calidad de docentes es buena y $18,3 \%$ indicaron excelente, la tendencia se manifiesta en los egresados de universidades públicas y privadas; el $59,3 \%$ de egresados de universidades privadas refieren que la calidad de sus profesores es buena, 25,9\% excelente, $26,4 \%$ percibe la actualización de sus profesores es excelente.

Según la SUNEDU (2018) hasta el año 2015 el 40\% de los docentes universitarios eran doctores, $40 \%$ con grado magister, $16 \%$ con título profesional y $5 \%$ bachiller, hace referencia que las instituciones educativas de nivel superior se están ajustando a lo estipulado por la Ley $\mathrm{N}^{\circ} 30220$ que hace referencia a que la enseñanza en dichas instituciones solo lo deben desarrollar los profesionales con grado de Magister como mínimo.

Otro aspecto a mencionar con respecto a la Ley $\mathrm{N}^{\circ} 30220$ la

\section{Recibido:23/08/19 Aprobado:02/09/19}


universidad debe garantizar al estudiante las herramientas, equipos y ambientes necesarios para poder desarrollar sus capacidades profesionales, por ende solicitar lo que necesite para su formación.

El docente tiene gran responsabilidad en la formación profesional de los estudiantes, quien haciendo uso de sus conocimientos, metodologías de enseñanza y de herramientas didácticas, esta última proporcionada por la entidad educativa, sobre todo en especialidades médicas que necesitan de imágenes que permitan visualizar cada parte de la anatomía humana.

En nuestra población de estudio evidenciamos que las calificaciones de los estudiantes de Facultad de Ciencias Médicas Universidad Nacional "Santiago Antúnez de Mayolo" no son destacadas y nuestra sospecha radica en las estrategias de enseñanza que se aplican los docentes, por ello los instrumentos que aplicaremos nos brindara detalles del nivel de incidencia.

\section{Antecedentes}

Es importante detallar estudios anteriores al nuestro como los de Castañeda (2015) quien se planteó como objetivo de investigar el valor de una intervención didáctica orientada al estímulo de competencias del área Biológica. En las conclusiones se destaca que se lograron establecer los indicadores de logro y sus niveles para cada una de las competencias formuladas, a través del análisis de los procesos que realizan los estudiantes, para lograr un nivel en la competencia, acorde a su recorrido en la carrera, además señala que se logró el diseño de las estrategias didácticas orientadas al desarrollo de las competencias.

Por otro lado, encontramos la investigación de Curvelo (2016) quien se planteó el objetivo de proponer un plan de estrategias didácticas para el logro del Aprendizaje Significativo en los alumnos de la población en estudio. En las conclusiones se destaca que para los docentes es importante revisar el contenido programático y con base a los contenidos, ver cuáles son las que más se adaptan a ellos, indicando algunos ejemplos como debate, demostraciones, instrucción personalizada. Además, agrega que los docentes, no realizan una planificación diaria de las actividades a desarrollar durante las clases que imparten, en consecuencia, no se planifica las estrategias metodológicas que se van a poner en práctica. En cuanto a las estrategias didácticas, los docentes usan el método de casos, el aprendizaje basado en problema, el método de proyectos y la técnica del debate, se puede concluir que solo utiliza durante el proceso de enseñanza aprendizaje estrategias tales como el aprendizaje basado en problemas, pero no está siendo uso del método de casos, el método de proyectos y la técnica del debate.

El objetivo principal de la investigación es conocer la relación entre las estrategias de enseñanza con el aprendizaje de la asignatura de Anatomía Humana de los estudiantes del III ciclo, Facultad de Ciencias Médicas Universidad Nacional "Santiago Antúnez de Mayolo" - Huaraz, 2018.

\section{MATERIALES Y MÉTODOS}

Con respecto a las finalidades de la investigación se consideró el enfoque cuantitativo de nivel explicativo con el objetivo de conocer la relación de las variables Estrategias de Enseñanza y la Aprendizaje de la Asignatura de Anatomía Humana, para la cual se aplicó un cuestionario y test de conocimientos, respectivamente.

Es importante detallar que los resultados de esta investigación fueron en base a la aplicación de los instrumentos.

\section{RESULTADOS}

La investigación se apoyó del tratamiento estadístico, para ello se consideró el programa SPSS, con el cual se estableció la contratación de las hipótesis, los cuales se muestran a continuación:

\section{Prueba de hipótesis general}

Ha. Las Estrategias de Enseñanza se relaciona significativamente con el Aprendizaje de la Asignatura de Anatomía Humana de los estudiantes del I ciclo, Facultad de Ciencias Médicas Universidad Nacional "Santiago Antúnez de Mayolo"-Huaraz, 2018.

Ho. Las Estrategias de Enseñanza no se relaciona significativamente con el Aprendizaje de la Asignatura de Anatomía Humana de los estudiantes del I ciclo, Facultad de Ciencias Médicas Universidad Nacional "Santiago Antúnez de Mayolo"-Huaraz, 2018.

Tabla 1

Correlación de la hipótesis general

\begin{tabular}{llrr}
\hline \multicolumn{3}{c}{ Correlaciones } & \\
\hline & & $\begin{array}{c}\text { Aprendizaje de } \\
\text { la asignatura } \\
\text { de Anatomía } \\
\text { Humana }\end{array}$ \\
\hline Rho de & $\begin{array}{l}\text { Estrategias } \\
\text { de }\end{array}$ & $\begin{array}{c}\text { Coeficiente de } \\
\text { correlación }\end{array}$ & 0,845 \\
enseñanza & Sig. (bilateral) & 0,000 \\
& & $\mathrm{~N}$ & 83 \\
\hline
\end{tabular}

De acuerdo a la fórmula de Rho, se halló un coeficiente de correlación muy buena de $\mathrm{Rho}=0,845$ y el $p=0,000$. Por lo cual se evidencia un rechazo a la hipótesis nula y la aceptación de la hipótesis alterna, se basa en lo señalado por la muestra que evidenció que las estrategias de enseñanzas aplicadas con una frecuencia de a veces, está relacionado al regular Aprendizaje de la Asignatura de Anatomía Humana.

\section{Contrastación de la primera hipótesis específica}

$\mathrm{H} 1$. La Activación de los conocimientos previos se relaciona significativamente con el Aprendizaje de la Asignatura de Anatomía Humana.

Ho. La Activación de los conocimientos previos no se relaciona significativamente con el Aprendizaje de la Asignatura de Anatomía Humana.

\section{Tabla 2}

Correlación de la primera hipótesis especifica

\begin{tabular}{llcr}
\hline & \multicolumn{3}{c}{ Correlaciones } \\
\hline & \multicolumn{3}{c}{$\begin{array}{c}\text { Aprendizaje de la asignatura de } \\
\text { Anatomía Humana }\end{array}$} \\
\hline & $\begin{array}{l}\text { Activación } \\
\text { Rho de } \\
\text { Spearman } \\
\text { conocimientos } \\
\text { previos }\end{array}$ & $\begin{array}{l}\text { Coeficiente de } \\
\text { correlación }\end{array}$ & 0,497 \\
& Sig. (bilateral) & 0,000 \\
\hline
\end{tabular}

Se determinó una correlación moderada de Rho $=0,497$ y el $p$ $=0,000$. Representa el rechazo a la hipótesis nula y la aceptación de la hipótesis alterna, cuyos resultados se basa en lo señalado por la muestra que evidenció que la activación de conocimientos previos aplicado con una frecuencia de a veces, está relacionado al regular aprendizaje de la asignatura de anatomía humana. 


\section{Contrastación de la segunda hipótesis específica}

H2. La orientación y guía de contenidos se relaciona significativamente con el aprendizaje de la asignatura de anatomía humana

Ho. La orientación y guía de contenidos no se relaciona significativamente con el aprendizaje de la asignatura de anatomía humana

Tabla 3

Correlación de la segunda hipótesis especifica

\begin{tabular}{llrr}
\hline & Correlaciones & \\
\hline & & $\begin{array}{l}\text { Aprendizaje de } \\
\text { la asignatura } \\
\text { de Anatomía } \\
\text { Humana }\end{array}$ \\
\hline $\begin{array}{l}\text { Rho de } \\
\text { Spearman }\end{array}$ & $\begin{array}{l}\text { Activación } \\
\text { de } \\
\text { conocimientos } \\
\text { previos }\end{array}$ & $\begin{array}{l}\text { Coeficiente de } \\
\text { correlación }\end{array}$ & 0,497 \\
& Sig. (bilateral) & 0,000 \\
\hline
\end{tabular}

Interpretación: La fórmula de Rho de Spearman, con un margen de error al $5 \%$, identificó un coeficiente de correlación moderada de $R h o=0,589$ y el $p=0,000$. Representa el rechazo a la hipótesis nula y la aceptación de la hipótesis alterna, cuyos resultados se basa en lo señalado por la muestra que encontró que la Orientación y guía de contenidos aplicadas con una frecuencia de a veces, está relacionado al regular Aprendizaje de la Asignatura de Anatomía Humana.

\section{Contrastación de la tercera hipótesis específica}

H3. La Codificación de la información se relaciona significativamente con el aprendizaje de la asignatura de anatomía humana

Ho. La Codificación de la información no se relaciona significativamente con el aprendizaje de la asignatura de anatomía humana

Tabla 4

Correlación de la tercera hipótesis especifica

\begin{tabular}{llrr}
\hline & Correlaciones & \\
\hline Spearman & $\begin{array}{l}\text { Aprendizaje de } \\
\text { la asignatura } \\
\text { de Anatomía } \\
\text { Humana }\end{array}$ \\
\hline $\begin{array}{l}\text { Rho de } \\
\text { Spearmación } \\
\text { información }\end{array}$ & $\begin{array}{l}\text { Codificaciónte de } \\
\text { correlación }\end{array}$ & 0,595 \\
\hline & Sig. (bilateral) & 0,000 \\
\hline
\end{tabular}

Se encontró un coeficiente de correlación moderada de Rho = 0,595 y el $p=0,000$. Por ello, se rechaza la hipótesis nula y se acepta la hipótesis alterna, cuyos resultados se basa en lo señalado por la muestra que encontró que la codificación de la información aplicadas con una frecuencia de a veces, está relacionado al regular aprendizaje de la asignatura de anatomía humana.

\section{Contrastación de la cuarta hipótesis específica}

H4. La organización de la información se relacionan significativamente con el Aprendizaje de la Asignatura de Anatomía Humana

Ho. La organización de la información no se relacionan significativamente con el aprendizaje de la asignatura de anatomía humana
Tabla 5

Correlación de la cuarta hipótesis especifica

\begin{tabular}{llcr}
\hline & Correlaciones & \\
\hline & & $\begin{array}{c}\text { Aprendizaje de } \\
\text { la asignatura } \\
\text { de Anatomía } \\
\text { Humana }\end{array}$ \\
\hline $\begin{array}{l}\text { Rho de } \\
\text { Spearman }\end{array}$ & $\begin{array}{l}\text { Organización } \\
\text { de la } \\
\text { información }\end{array}$ & $\begin{array}{c}\text { Coeficiente de } \\
\text { correlación }\end{array}$ & 0,581 \\
& Sig. (bilateral) & 0,000 \\
\hline
\end{tabular}

Se determinó una correlación moderada de Rho $=0,581$, se rechaza la hipótesis nula y se acepta la hipótesis alterna, en referencia lo manifestado por la muestra se establece que la Organización de la información aplicadas con una frecuencia de a veces, está relacionado al regular Aprendizaje de la Asignatura de Anatomía Humana.

\section{Contrastación de la quinta hipótesis específica}

H5. El Enlace de conocimientos previos y nuevos se relacionan significativamente con el Aprendizaje de la Asignatura de Anatomía Humana Ho. El Enlace de conocimientos previos y nuevos no se relacionan significativamente con el Aprendizaje de la Asignatura de Anatomía Humana.

Tabla 6

Correlación de la quinta hipótesis especifica

\begin{tabular}{llrr}
\hline & \multicolumn{3}{c}{ Correlaciones } \\
\hline & \multicolumn{3}{c}{$\begin{array}{c}\text { Aprendizaje de la asignatura de } \\
\text { Anatomía Humana }\end{array}$} \\
\hline Rho de & $\begin{array}{l}\text { Enlace de } \\
\text { conocimientos } \\
\text { Spearman y } \\
\text { nuevos }\end{array}$ & $\begin{array}{l}\text { Coeficiente de } \\
\text { correlación }\end{array}$ & 0,728 \\
& Sig. (bilateral) & 0,000 \\
\hline
\end{tabular}

Interpretación: La aplicación de la fórmula de Rho de Spearman, margen de error al $5 \%$, se halló una correlación buena de $\mathrm{Rho}=0,728$ y el $p=0,000$. Representa el rechazo a la hipótesis nula y la aceptación de la hipótesis alterna, cuyos resultados se basa en lo señalado por la muestra que evidenció que el enlace de conocimientos previos y nuevos aplicadas con una frecuencia de a veces, está relacionado al regular aprendizaje de la asignatura de anatomía humana.

\section{DISCUSIÓN}

La hipótesis general halló una correlación muy buena de Rho = 0,845 , con respecto a las variables Estrategias de Enseñanza y Aprendizaje de la Asignatura de Anatomía Humana, cuyos resultados se deben a que en la primera variable la mayoría de los estudiantes representados en el $74,7 \%$ lo identifican como a veces, seguido del $18,1 \%$ como siempre; incide en la segunda variable cuyos aprendizajes están en el nivel regular por el $79,5 \%$, mientras que el $12,0 \%$ en el nivel bueno. Concluimos que la frecuencia con la cual se aplican estrategias de enseñanza no es suficiente para la mayoría de estudiantes las calificaciones en el curso de Anatomía Humana no son destacadas perjudicando así su aprendizaje.

La hipótesis específica 1 estableció una correlación moderada de $R$ ho $=0,497$, con respecto a la dimensión activación de conocimientos previos y la variable aprendizaje de la asignatura de Anatomía Humana, cuyo análisis se debe a que en la primera dimensión la mayoría de los estudiantes 
expresaron $56,6 \%$ lo identifican como a veces, seguido del $27,7 \%$ como siempre; lo cual está relacionada a lo encontrado en la segunda variable cuyos aprendizajes están en el nivel regular por el $79,5 \%$, mientras que el $12,0 \%$ en el nivel bueno. En definitiva, los estudiantes no están conforme con la frecuencia con la cual los docentes hacen uso de estrategias para introducir temas, explicar metodologías, respetar puntos de vista, promover participación, entre otros, por ello se considera insuficiente para poder mejorar sus calificaciones en el curso de Anatomía Humana.

La hipótesis específica 2 identificó una correlación moderada de $R$ ho $=0,589$, en base a la dimensión Orientación y guía de contenidos, y la variable aprendizaje de la asignatura de Anatomía Humana, cuyo resultado se debe a que la segunda dimensión es verificada mayoritariamente de a veces por un $63.9 \%$, seguido de siempre con $28,9 \%$; lo cual genera incidencias con respecto a la segunda variable que hace referencia mayoritaria en el nivel regular por el $79,5 \%$, continuando con el nivel bueno con un 12,0\%. En consecuencia, los estudiantes no están conforme con la frecuencia con la cual los docentes hacen uso de estrategias para enfatizar contenidos relevantes, identificar conceptos y confrontar posturas, en otras palabras, se considera insuficiente para el mejoramiento de sus calificaciones en el curso de Anatomía Humana.

La hipótesis específica 3 encontró una correlación moderada de $R$ ho $=0,595$, con respecto a la dimensión codificación de la información y la variable aprendizaje de la asignatura de Anatomía Humana, debido a que la tercera dimensión es considerada por la mayoría como a veces por un $60,2 \%$, seguido de $28,9 \%$ como siempre; está relacionada a los aprendizajes de la segunda variable hace referencia mayoritaria en el nivel regular por el $79,5 \%$, continuando con el nivel bueno con un $12,0 \%$. En suma, los estudiantes no consideran totalmente satisfactorio la frecuencia con la cual los docentes hacen uso de estrategias para ilustrar la información, usar gráficos, realizar preguntas, por ello no ayuda a mejorar las calificaciones del curso de Anatomía Humana.

La hipótesis específica 4 estableció una correlación moderada de $R$ ho $=0,581$, que incluye a la dimensión organización de la información y la variable aprendizaje de la asignatura de anatomía humana, evidenciado por que la cuarta dimensión es referida de a veces por el $66,3 \%$, seguido de siempre con el $19,3 \%$; ocasionando incidencias en la segunda variable que alcanzan promedios mayoritarios de regular en el $79,5 \%$, lejos del $12,0 \%$ como bueno. Por consiguiente, las perspectivas académicas de los estudiantes se encuentran insatisfechos en cuanto al uso de estrategias del docente para elaborar resúmenes, mapas conceptuales y planear actividades, por ello no ayuda a mejorar las calificaciones del curso de Anatomía Humana.

La hipótesis específica 5 halló una correlación buena de Rho $=$ 0,728 , con respecto a la dimensión Conocimientos previos y nuevos, y la variable Aprendizaje de la Asignatura de Anatomía Humana, lo cual establece que la quinta dimensión es mayoritariamente considerada de a veces por el 69,9\%, seguido de un $18,1 \%$ calificado de siempre; cuyas apreciaciones están relacionadas a los aprendizajes a la segunda variable que hace referencia mayoritaria en el nivel regular por el $79,5 \%$, continuando con el nivel bueno con un $12,0 \%$. Entonces, los estudiantes no están conforme con la frecuencia que los docentes hacen uso de estrategias para Organizar información previamente, textos narrativos e informativos, y la variedad de textos, se considera insuficiente para poder mejorar sus calificaciones en el curso de Anatomía Humana.

Comparando estos resultados con los de Membreño (2017) evidenciamos la necesidad docente de mejorar sus capacidades metodológicas debido a que los constantes avances del curso a dictar, los conocimientos son constantes y la complejidad del mismo se convierte en un motivo más para ir innovando estrategias, resultados de similares características hemos podido evidenciarlo ya que la forma como se enseña es fundamental para que los estudiantes aprendan la terminología científicas y otras necesidades académicas que si no se apoya de estrategias se reduce las posibilidades de mejorar sus aprendizajes.

La importancia y la necesidad de garantizar aprendizajes según Salazar (2016) se debe al establecimiento idóneo de una metodología de enseñanza, sin embargo, alerta que se necesita un seguimiento constante para identificar si se están alcanzando los objetivos académicos, estas conclusiones se comparan al nuestro en el sentido de la necesidad establecer metodologías para mejorar aprendizajes, los cuales deberán ser reflejado en sus calificaciones.

\section{AGRADECIMIENTOS}

En primer lugar doy infinitamente gracias a Dios, por haberme dado fuerza y valor para culminar esta etapa de mi vida.

Agradezco a mis padres, que sin duda alguna en el trayecto de mi vida me han demostrado su amor, y su apoyo.

\section{REFERENCIAS BIBLIOGRÁFICAS}

Castañeda, L. (2015). Enseñanza de la anatomía orientada al desarrollo de competencias en la carrera de Bioingeniería (Tesis Posgrado) Universidad Nacional del Litoral, Argentina. Recuperado en: http://bibliotecavirtual.unl.ed u.ar:8080/tesis/bitstream/ha ndle/11185/770/Tesis. pdf? s equence=1\&isAllowed=y

Curvelo, D. (2016). Estrategias didácticas para el logro del aprendizaje significativo en los alumnos cursantes de la asignatura seguridad industrial (Tesis Posgrado) Universidad de Carabobo, Venezuela. Recuperado en: h t t p: / / r i u c.b c.u c . e d u. ve / b i t s t r eam/123456789/3878/1/dcu rvelo.pdf

INEI (2015). Encuesta Nacional a Egresados Universitarios y Universidades, 2014. Lima: Instituto $\mathrm{Nacional}$ de Estadística e Informática. Recuperado en: https://www.inei.gob.pe/med ia/MenuRecursivo/publicaci ones digitales/Est/Lib1298/Libro.pdf

Ley $N^{\circ}$ 30220. Diario Oficial El Peruano, Lima, Perú, 9 de julio de 2014.

Membreño, A. (2017). Análisis de las estrategias de enseñanza que utilizan los docentes y las estrategias de aprendizaje de los estudiantes del Grupo No HAB-7119-112016 del primer nivel de inglés, en el primer turno diurno, en el Tecnológico Nacional de Idiomas, durante el segundo semestre del año académico, $2016 \quad$ ( T e s i s Posgrado) Universidad Nacional Autónoma de Nicaragua, $\mathrm{N}$ i c a r a g u a. R e c u p e r a d o e $n$ : http://repositorio.unan.edu.ni/4603/1/96828.pdf

Salazar, R. (2016). Aprendizaje asistido por pares cercanos como estrategia para el desarrollo de un curso de anatomía humana de abdomen (Tesis Posgrado) Universidad Complutense de Madrid, E s p a ñ a $\mathrm{R}$ e c u p e $\mathrm{r}$ a d o e $\mathrm{n}$ : https://eprints.ucm.es/38564/1/T37546.pdf

SUNEDU (2018). Informe bienal sobre la realidad universitaria peruana. Lima: Superintendencia Nacional de Educación Superior Universitaria. 